\title{
Retroperitoneal Laparoscopic Nephroureterectomy for Tuberculous Nonfunctioning Kidneys: a single-center experience
}

\author{
Xiquan Tian ${ }^{1}$, Mingshuai Wang ${ }^{1}$, Yinong Niu ${ }^{1}$, Junhui Zhang ${ }^{1}$, Liming Song ${ }^{1}$, Nianzeng Xing ${ }^{1}$ \\ ${ }^{1}$ Department Of Urology, Affiliated Beijing Chao-Yang Hospital Of Capital Medical University, Beijing, China
}

\section{ABSTRACT}

Purpose: To present our surgical techniques and experiences of retroperitoneal laparoscopic nephroureterectomy for the treatment of tuberculous nonfunctioning kidneys. Materials and Methods: From March 2005 to March 2013, a total of 51 patients with tuberculous nonfunctioning kidney underwent retroperitoneal laparoscopic nephroureterectomy at our medical center. The techniques included early control of renal vessels and dissection of the diseased kidney along the underlying layer outside the Gerato's fascia. The distal ureter was dissected through a Gibson incision and the entire specimen was removed en bloc from the incision. Patient demographics, perioperative characteristics and laboratory parameters as well as postoperative outcome were retrospectively reviewed.

Results: Retroperitoneal laparoscopic nephroureterectomy was successfully performed in 50 patients, whereas one case required conversion to open surgery due to non-progression of dissection. The mean operating time was 123.0 minutes (107-160 minutes) and the mean estimated blood loss was $134 \mathrm{~mL}(80-650 \mathrm{~mL})$.The mean postoperative hospital stay was 3.6 days (3-5days) and the mean return to normal activity was 11.6 days (10-14days). Most intra-operative and post-operative complications were minor complications and can be managed conservatively. After 68 months (12-96 months) follow-up, the outcome was satisfactory, and ureteral stump syndrome did not occur. Conclusions: Retroperitoneal laparoscopic nephroureterectomy as a minimally invasive treatment option is feasible for treatment of tuberculous nonfunctioning kidneys.

\section{ARTICLE INFO}

Key words:

Tuberculosis; Laparoscopy;

Nephroureterectomy; Kidney

Int Braz J Urol. 2015; 41: 296-303

Submitted for publication:

March 09, 2014

Accepted after revision:

June 23, 2014

\section{INTRODUCTION}

Since the initial introduction of laparoscopic nephrectomy in 1991 (1), laparoscopic surgery has made tremendous progress in the urological field. Currently, many complex kidney surgeries such as radical nephrectomy, partial nephrectomy and living donor nephrectomy can be successfully completed by laparoscopic approach, and the safety and effectiveness have been confirmed by several studies $(2,3)$. However, laparoscopic nephroureterectomy for tuberculous nonfunctioning kidney has always been a highly challenging procedure because of significant perinephric and perihilum inflammation and severe adhesion with adjacent structures. To the best of our knowledge, several small case series were reported on the outcomes and feasibility of laparoscopic nephroureterectomy for patients with tuberculous nonfunctioning kidneys (4-8). It is still difficult to 
interpret the overall risk of laparoscopic nephroureterectomy for tuberculous nonfunctioning kidney due to the lack of cases in these reports, and large trials are required to draw definitive conclusions. Moreover, few studies dived into whether it is necessary to remove the distal ureter in the laparoscopic nephroureterectomy procedure for the treatment of nonfunctioning tuberculous kidneys.

In the present study, we aim to report our eight years' experience with retroperitoneal laparoscopic nephroureterectomy for the management of tuberculous nonfunctioning kidneys.

\section{MATERIALS AND METHODS}

Patients who underwent retroperitoneal laparoscopic nephroureterectomy with unilateral nonfunctioning kidney secondary to tuberculosis were included in the study. Patients with a diseased kidney less than $10 \mathrm{~cm}$ in diameter and without surgical history at the same anatomical site were selected for this surgery. Patients were excluded from the study if the final histopathologic examination demonstrated that the diseased kidneys were not associated with tuberculosis. From March 2005 to March 2013, a total of 51 patients were selected for this study. This study was approved by the Ethics Committee of Beijing Chao-Yang Hospital, affiliated with Capital Medical University. The advantages and risks of the laparoscopic surgery and the possibility of conversion to open surgery were fully explained to patients; all patients signed a written consent form before surgery.

The most common clinical presentation was irritative voiding symptoms (23 cases), followed by recurrent urinary tract infection (18 cases), gross hematuria (6 cases), ipsilateral flank pain ( 3 cases) and scrotal mass (1 case). Renal tuberculosis was suspected based on symptoms and was confirmed by a positive urine smear, urine polymerase chain reaction for acid-fast bacilli and histopathologic evaluation of bladder biopsy. A computerized tomography was used to demonstrate imaging manifestations of the entire urinary tract. A renal nuclear scan was utilized to evaluate glomerular filtration rates of the diseased kidney and contralateral kidney. Every patient had nor- mal or mild impaired function in the contralateral kidney, and the glomerular filtration rate of the diseased side was less than $10 \mathrm{~mL} / \mathrm{min} / 1.73 \mathrm{~m}^{2}$. All patients initially received an anti-tuberculous remedy for 4 weeks to 3 months before operation. The standard regimen was isoniazid $5 \mathrm{mg} / \mathrm{kg}$ orally once daily, rifampicin $10 \mathrm{mg} / \mathrm{kg}$ orally once daily and ethambutol $15 \mathrm{mg} / \mathrm{kg}$ orally once daily. Symptoms of active tuberculosis, such as fever, night sweats, anemia, anepithymia and a rapid erythrocyte sedimentation rate, were controlled before operations. To avoid data deviations due to surgical learning curve, all surgeries were performed by the same surgeon (Dr. Nianzeng Xing). The intra-operative complications were analyzed using Satava classification (9), and the postoperative complications were graded by modified Clavien classifications (10).

All patients were regularly followed up at the second week, third month and sixth month after discharge and every 6 months thereafter till five years. A physical examination, hematological examination and ultrasonography were done during regular follow-up. An intravenous urography, renal scan and cystography were performed according to above examinations. To ensure maximal chance of bacteriologic cure, all patients received at least 6 months of antituberculous treatment after surgery.

\section{OPERATION TECHNIQUE}

Patients were placed in the lateral flank position with elevation of the diseased kidney bridge after general anesthesia. All surgical procedures were conducted as a retroperitoneoscopic radical nephrectomy, and four trocars were employed (6, 11, 12) (Figure-1). A $2 \mathrm{~cm}$ incision was made $2 \mathrm{~cm}$ below the costal margin on the posterior axillary line. Using a forceps to bluntly penetrate the muscle layer and lumbodorsal fascia to the retroperitoneal space and an index finger to dilate the space, an enough retroperitoneal space was created to hold an uninflated balloon. Then the balloon was inflated with $1000 \mathrm{~mL}$ air and kept for 3 minutes. A $5 \mathrm{~mm}, 12 \mathrm{~mm}$, and $10 \mathrm{~mm}$ trocar were placed respectively at the anterior axillary line around the 11 th rib tip, $2 \mathrm{~cm}$ inside the anterior superior 
Figure 1 - Positions of the four working trocars.

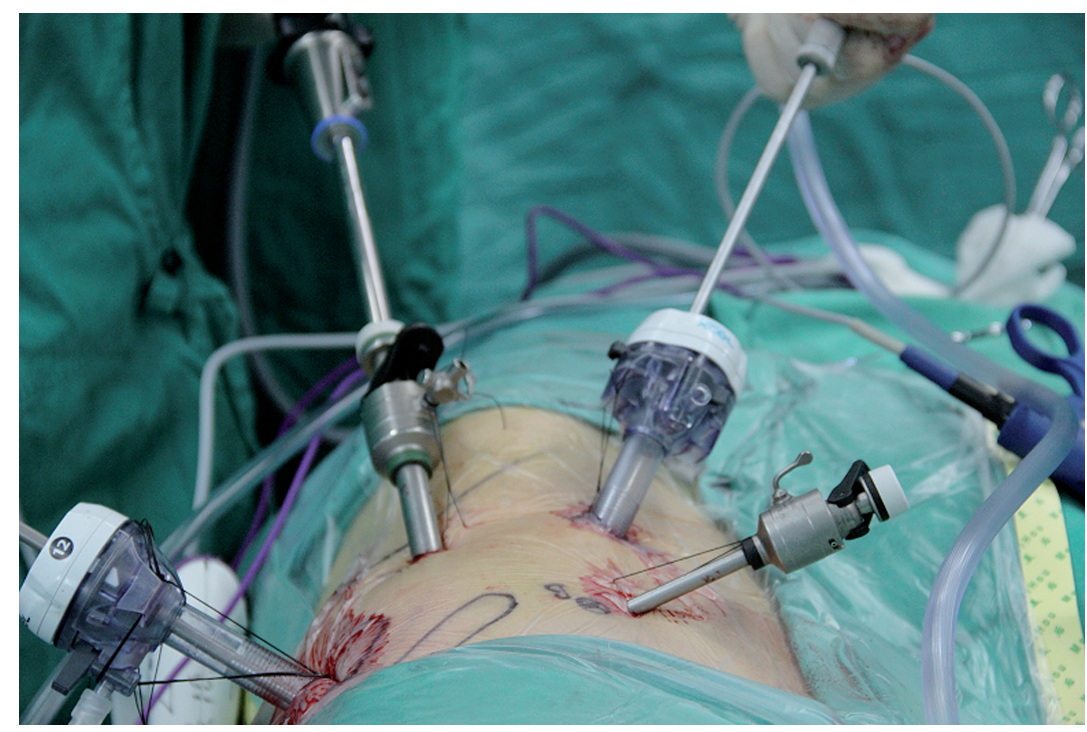

iliac spine, and 2-cm above the iliac crest for the $30^{\circ}$ Olympus laparoscope. Finally, a $12 \mathrm{~mm}$ trocar was placed in the initial hole. The Gerota's fascia was cut horizontally from the front edge of the psoas muscle up to the diaphragm and down to the lower pole of the kidney, and the renal hilum was accessed by subtle but sharp dissection towards the renal artery pulsation. The renal artery and vein were successively ligated with three Hem-0-Lock clips and dissected (Figure-2). The kidney was subsequently mobilized outside Gerota's fascia as far as possible using sharp dissection and

Figure 2 - The renal artery (RA) and renal vein (RV) were clipped with Hem-0-Iok clips and divided by LigaSure.

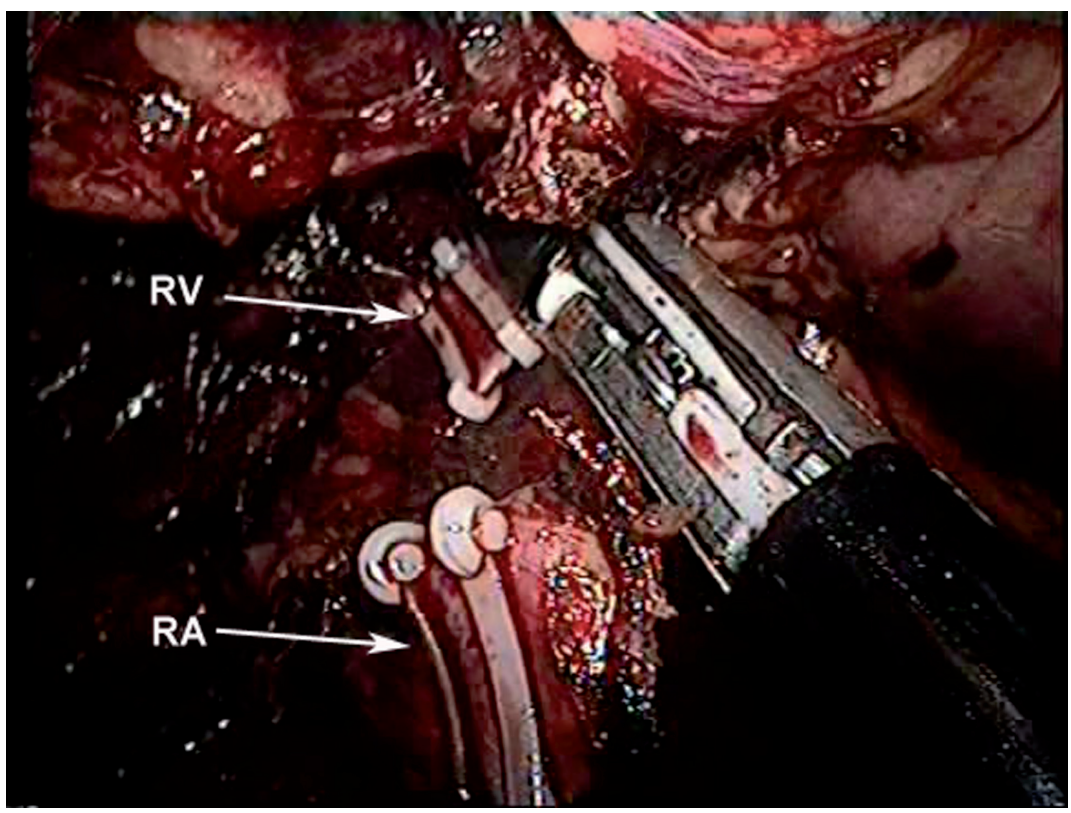


care was taken to avoid puncturing the kidney sac (Figure-3). The ureter was then identified and dissected as distally as possible at which point it is clipped with a Hem-0-Lock clip and left in situ. The retroperitoneal space was inspected with in- age from 23 to 78 years old. Totally, 30 diseased kidneys were at the left side and 21 cases at the right side. The mean disease course was 7.3 months (range: 3-21 mon). Of 51 patients, 6 cases with contracted bladder had mild to moderate re-

Figure 3 - Dissection of the diseased kidney outside the Gerato's fascia.

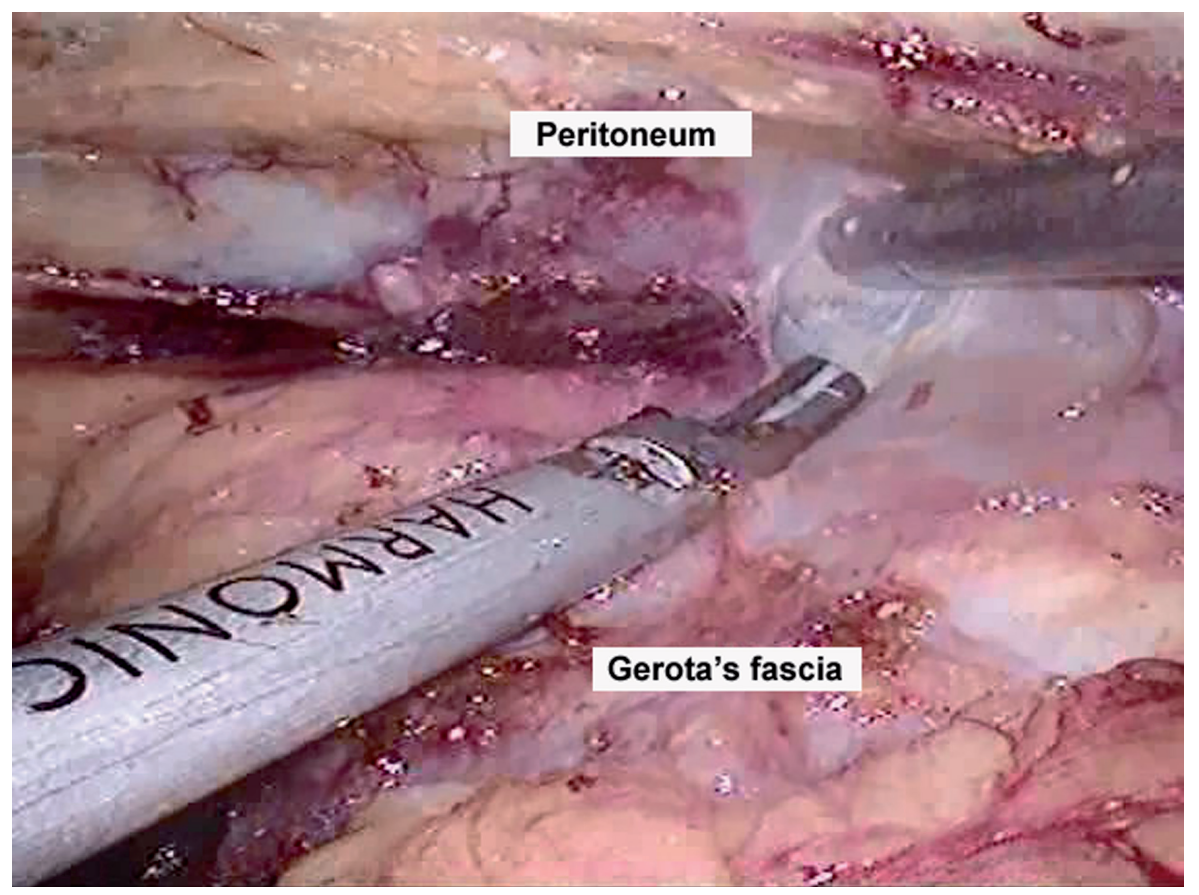

sufflation pressure at $5 \mathrm{mmHg}$ to ensure adequate homeostasis. The trocar incisions were closed and the patient was changed to supine position. An approximately 7-8 $\mathrm{cm}$ long Gibson incision was made in the lower abdominal region. The distal ureter was gently dissected until some of the intramural ureter was mobilized, and then the bladder cuff was cut to free the specimen. After the bladder incision was totally sutured, the entire kidney within Gerota's fascia and ureter were removed en bloc from the Gibson incision.

\section{RESULTS}

The demographic profiles and perioperative parameters of patients are shown in Table-1. There were 22 males and 29 females, ranging in nal function impairment (range of serum creatinine level: 189-450 $\mathrm{mmol} / \mathrm{L}$ ) and required sigmoid augmentation cystoplasty.

Retroperitoneal laparoscopic nephroureterectomy was successfully completed in 50 patients. One patient required conversion to open surgery due to lack of progress in dissection, and the open nephroureterectomy was successfully performed. The mean operating time was 123.0 minutes (range: 109$160 \mathrm{~min})$. The mean estimated blood loss was 134 $\mathrm{mL}$ (range, 80-650 $\mathrm{mL}$ ) and two patients required intraoperative transfusion of $2 \mathrm{U}$ packed red blood cells. The mean hospital stay was 3.6 days (range: 3-5 days) and the time of returning to normal activity was 11.6 days varying from 10 to 14 days.

The intra-operative complications analyzed using Satava classification are shown in Table-2. 
Table 1 - Preoperative and operative characteristics of patients.

\begin{tabular}{lc}
\hline Parameter & Value (range) \\
\hline Age (years) & $39.3(23-78)$ \\
Sex (M/F) & $22 / 29$ \\
Side (right/left) & $21 / 30$ \\
BMI (kg/m²) & $24.3(21-27)$ \\
Disease course (months) & $7.3(3-21)$ \\
Operative time (minutes) & $123(109-160)$ \\
Estimated Blood loss (mL) & $134(80-650)$ \\
Blood transfusion (n) & 2 \\
Hospital stay (days) & $3.6(3-5)$ \\
Conversion (n) & 1 \\
Return to previous activities(days) & $11.6(10-14)$ \\
Follow-up time (months) & $68(12-96)$ \\
\hline
\end{tabular}

The most common complication was peritoneal tear (7.84\%), which can be managed laparoscopically with no need to conversion. Renal venous injury $(1.96 \%)$ occurred in one patient due to severe adhesion. However, the bleeding was successfully controlled and did not hamper the successful completion of the laparoscopic procedure. One diseased kidney was inadvertently punctured $(1.96 \%)$ during dissection. The working space was thoroughly washed with a copious amount of normal saline plus streptomycin at the end of the pro-
Table 2 - Peri-operative complication according to Satava classification system.

\begin{tabular}{ll}
\hline & No. of cases \\
\hline $\begin{array}{l}\text { Satava grade I (incidents without } \\
\text { consequence) }\end{array}$ & $1(1.96 \%)$ \\
$\quad$ Sinus arrhythmia & \\
Satava grade II (incidents repaired \\
intraoperatively) \\
$\quad \begin{array}{l}\text { Renal vein injury } \\
\quad \text { Lumbar vein injury }\end{array}$ & $1(1.96 \%)$ \\
$\quad$ Peritoneal injury & $4(7.84 \%)$ \\
$\quad$ Spillage of pus & $1(1.96 \%)$ \\
$\begin{array}{l}\text { Satava grade III (incidents requiring open } \\
\text { conversion) }\end{array}$ & $1(1.96 \%)$ \\
\hline
\end{tabular}

cedure, and no disseminated or systemic disease was identified during long-term follow-up. The postoperative complications graded by modified Clavien classifications are shown in Table-3. The grade I and II complications were 19.6\% in all. One patient was reoperated for a hematoma in retroperitoneum, which was a grade III complication $(1.96 \%)$.

The histopathologic examination confirmed the diagnosis of renal tuberculosis in all patients. The common microscopic findings were

Table 3 - Postoperative complications stratified according to the modified Clavien classification.

\begin{tabular}{llc}
\hline Clavien Grade & $n$ & Management \\
\hline Grade I (11.76\%) & 6 & Antipyretics \\
Fever & 1 & Observation \\
Transient elevation in creatinine & 2 & Observation \\
Subcutaneous emphysema & 3 & Blood transfusion \\
Grade II (7.84\%) & 4 & antibiotics \\
Blood loss anemia & 2 & Wound opening and antibiotics \\
Pneumonia & 1 & Re-operation \\
Wound infection & 1 & 1 \\
Grade III (1.96\%) & 1 &
\end{tabular}


tuberculous pyelonephritis with caseation, perinephritis and tuberculous pyonephrosis. Similar changes were found in the walls of ureters. The normal tissue was completely or partly replaced by caseous substance or fibrous tissue, and the ureter became thicken and stenotic.

After 68 months (range: 12-96 months) regular follow-up, the outcomes were satisfactory. All patients achieved improvement in symptoms and renal function. No local or disseminated recurrence was identified (evaluated by urine polymerase chain reaction for acid-fast bacilli, hematologic examination and imaging examination). The ureteral stump syndrome did not occur in our patients.

\section{DISCUSSION}

Benign kidney diseases are the most common indications for laparoscopic nephroureterectomy (13). However, laparoscopic nephroureterectomy for inflammatory renal conditions such as tuberculous nonfunctioning kidney, long-standing renal stone disease and xanthogranulomatous pyelonephritis has always been considered as a relative contraindication because of higher rate of complications and conversion to open surgery. In this study, we retrospectively reviewed 51 patients with tuberculous nonfunctioning kidneys managed by retroperitoneal laparoscopic nephroureterectomy in respect to surgical techniques, perioperative outcome and follow-up, and the results are encouraging.

In our cases, we performed all surgeries by retroperitoneal laparoscopic approach for we have high volume of experience with this approach (12). Current researches have demonstrated that the laparoscopic operation for inflammatory nonfunctioning kidney can be accomplished through either the transperitoneal or the retroperitoneal approaches $(5,7)$, and the choice depends mainly on the surgeon's educational background and laparoscopic experiences. Although the retroperitoneal approach has some disadvantages such as the limited working space, difficulty to identify the anatomical structure as well as a longer learning curve compared to the transperitoneal approach, it facilitates to control renal vessels early and avoids contamination with the pus and potential injury to intraperitoneal organs (14).

Only a few small series have been reported addressing laparoscopic nephroureterectomy for tuberculous nonfunctioning kidneys. The reported rates of successive laparoscopic surgery were between $77.8 \%$ and 100\% (5-8). The largest cohort included only 31 patients (7). In the present study, a total of 51 patients were enrolled and laparoscopic nephroureterectomy was successfully performed in 50 patients (98.0\%) with only one conversion due to dense adhesions. The peri-operative data, including mean operative time, estimated blood loss, hospital stay and complications, were consistent with previous reports $(4,15,16)$. These data support that retroperitoneal laparoscopic nephroureterectomy is feasible to treat patients with nonfunctioning tuberculous kidneys.

Various technical modifications for laparoscopic nephrectomy in the setting of nonfunctioning tuberculous kidneys and other inflammatory conditions have been proposed to reduce technical difficulties. Early control of renal vessels is an essential step of this procedure which can substantially minimize widespread bleeding and facilitate subsequent dissection $(6,11)$. Dissection of renal pedicle vessels is extremely challenging because severe inflammatory process makes anatomy layers obliterated and distorted. En bloc ligation of renal hilar has been recommended to simplify the procedure for more difficult cases (17); however, such technique has been reported to be associated with the very rare complication of arteriovenous fistula formation, primarily in inflammatory renal pathologic features (18). Therefore, individual ligation of the renal artery and vein was strongly preferred in our cohort. A careful, slow, patient dissection is utmost important in this procedure. The pulsations of the abdominal aorta and renal artery, and the undulation of inferior vena cava are useful markers. The renal pedicle should be approached as close as possible to the inferior vena cava on the right side and aorta on the left side, and much difficulty was not encountered in the dissection of the fibrous tissue in this region. After ligation of renal vessels, it is crucial to identify an appropriate layer to intactly move the diseased kidney. It has been reported that the 
underlying layer outside the Gerota's fascia is less affected by inflammation, and a dissection along this layer offers better progression and far less bleeding (19-21). Subcapsular nephrectomy has also been described for the retroperitoneoscopic approach of LN in inflammatory conditions when dense adhesions were encountered $(22,23)$. For nonfunctioning tuberculous kidneys, we found that the adhesive bundles and fibrosis were mainly localized inside the Gerota's fasica after a minimum of 4 weeks of anti-tuberculous chemotherapy. Dissection can be performed with minimal difficulty by keeping the plane outside Gerota's fascia. If difficulty with laparoscopic dissection is encountered in one area, the procedure can be carried out in other areas. The surgeon should try to achieve as much dissection as possible. The safe layer is usually visualized in the dense adhesive areas once the diseased kidney has been mostly dissected. Moreover, the introduction of advance equipments, such as the harmonic scalpel, Ligasure and bipolar electric coagulation is an excellent alternative to avoid bothersome bleeding and makes surgeries easier.

Severe ureteral stump syndrome was reported after simple nephrectomy for treatment of nonfunctioning tuberculous kidneys, and powerful anti-tuberculous regimen failed to completely cure the severe irritative symptoms in these cases $(24,25)$. To ensure optimal effectiveness, it becomes apparent that total ureterectomy should be applied for treatment of nonfunctioning tuberculous kidneys. The final histopathological outcome of all patients in our study demonstrated that ureters were all diffusely invaded by tuberculosis, which supports the essentiality of total ureterectomy for nonfunctioning tuberculous kidneys. Multiple techniques have been used to manage the distal ureter during laparoscopic nephrouretectomy, including open incision and resection via an intravesical or extravesical approach, endoscopic, laparoscopic stapling of the bladder cuff, or combinations of several techniques. Considering the clinical-pathological characteristics of the tuberculosis and our experience, we recommend conventional open surgery to deal with the distal ureter. After completion of the laparoscopic procedure in our series, an approximately $7-8 \mathrm{~cm}$ long Gibson incision was made and the distal ureter was dissected till to the bladder wall, and the intact specimen was removed en bloc from the incision. This approach facilitates to remove more tuberculous tissue to eliminate the potentiality of ureteral stump syndrome.

There are several limitations in our study. First, our case number is relatively small. Second, this is a retrospective study of a single center. Randomized controlled trials with large sample size are needed to prove the feasibility and superiority of retroperitoneal laparoscopic approach to manage tuberculous nonfunctioning kidneys.

\section{CONCLUSIONS}

Our results indicated that retroperitoneal laparoscopic nephroureterectomy is a safe and effective method for the treatment of tuberculous nonfunctioning kidneys with satisfactory long-term follow-up outcomes. However, randomized controlled studies with large sample size and high-quality design are needed to confirm our results.

\section{CONFLICT OF INTEREST}

None declared.

\section{REFERENCES}

1. Clayman RV, Kavoussi LR, Soper NJ, Dierks SM, Meretyk S, Darcy MD, et al. Laparoscopic nephrectomy: initial case report. J Urol. 1991;146:278-82.

2. Fahlenkamp D, Rassweiler J, Fornara P, Frede T, Loening SA. Complications of laparoscopic procedures in urology: experience with 2,407 procedures at 4 German centers. J Urol. 1999;162:765-70; discussion 770-1.

3. Portis AJ, Yan Y, Landman J, Chen C, Barrett PH, Fentie $\mathrm{DD}$, et al. Long-term followup after laparoscopic radical nephrectomy. J Urol. 2002;167:1257-62.

4. Hemal AK, Gupta NP, Wadhwa SN, Goel A, Kumar R. Retroperitoneoscopic nephrectomy and nephroureterectomy for benign nonfunctioning kidneys: a single-center experience. Urology. 2001;57:644-9.

5. Chibber PJ, Shah HN, Jain P. Laparoscopic nephroureterectomy for tuberculous nonfunctioning kidneys compared with laparoscopic nephroureterectomy for other diseases. J Laparoendosc Adv Surg Tech A. 2005;15:308-11. 
6. Hemal AK, Gupta NP, Kumar R. Comparison of retroperitoneoscopic nephrectomy with open surgery for tuberculous nonfunctioning kidneys. J Urol. 2000;164:32-5.

7. Lee KS, Kim HH, Byun SS, Kwak C, Park K, Ahn H. Laparoscopic nephrectomy for tuberculous nonfunctioning kidney: comparison with laparoscopic simple nephrectomy for other diseases. Urology. 2002;60:411-4.

8. Zhang X, Zheng T, Ma X, Li HZ, Li LC, Wang SG, et al. Comparison of retroperitoneoscopic nephrectomy versus open approaches to nonfunctioning tuberculous kidneys: a report of 44 cases. J Urol. 2005;173:1586-9.

9. Satava RM. Identification and reduction of surgical error using simulation. Minim Invasive Ther Allied Technol. 2005;14:257-61.

10. Dindo D, Demartines N, Clavien PA. Classification of surgical complications: a new proposal with evaluation in a cohort of 6336 patients and results of a survey. Ann Surg. 2004;240:205-13.

11. Hemal AK, Mishra S. Retroperitoneoscopic nephrectomy for pyonephrotic nonfunctioning kidney. Urology. 2010;75:5858.

12. Ping $H$, Xing NZ, Zhang $J H$, Yan $Y$, Kang $N$, Niu YN. Application of the Hem-o-lok ligation system in laparoscopic nephrectomy. Surg Endosc. 2010;24:1494-7.

13. Raghuram S, Godbole HC, Dasgupta P. Laparoscopic nephrectomy: the new gold standard? Int J Clin Pract. 2005;59:128-9.

14. Gupta NP, Hemal AK, Mishra S, Dogra PN, Kumar R. Outcome of retroperitoneoscopic nephrectomy for benign nonfunctioning kidney: a single-center experience. J Endourol. 2008;22:693-8.

15. Arvind NK, Singh 0 , Ali Q, Gupta SS, Sahay S. Laparoscopic nephrectomy in xanthogranulomatous pyelonephritis: 7-year single-surgeon outcome. Urology. 2011;78:797-801.

16. Ivey BS, Lucas SM, Meyer CA, Emley TE, Bey A, Gardner TA, et al. Conversions in laparoscopic renal surgery: causes and outcomes. J Endourol. 2011;25:1167-73.

17. Kouba E, Smith AM, Derksen JE, Gunn K, Wallen E, Pruthi RS. Efficacy and safety of en bloc ligation of renal hilum during laparoscopic nephrectomy. Urology. 2007;69:226-9.
18. Lacombe M. Renal arteriovenous fistula following nephrectomy. Urology. 1985;25:13-6.

19. Duarte RJ, Mitre Al, Chambô JL, Arap MA, Srougi M. Laparoscopic nephrectomy outside gerota fascia for management of inflammatory kidney. $J$ Endourol. 2008;22:681-6.

20. Shekarriz B, Meng MV, Lu HF, Yamada H, Duh QY, Stoller ML. Laparoscopic nephrectomy for inflammatory renal conditions. J Urol. 2001;166:2091-4.

21. Tepeler A, Akman T, Tok A, Kaba M, Binbay M, Müslümanoğlu AY, et al. Retroperitoneoscopic nephrectomy for nonfunctioning kidneys related to renal stone disease.Urol Res. 2012;40:559-65.Erratum in: Urol Res. 2012;40:567.

22. Xu Z, Xin M, Hong-Zhao L, Zhong C, Li LC, Ye ZQ. Retroperitoneoscopic subcapsular nephrectomy for infective nonfunctioning kidney with dense perinephric adhesions. BJU Int. 2004;94:1329-31.

23. Kapoor R, Vijjan V, Singh K, Goyal R, Mandhani A, Dubey $D$, et al.Is laparoscopic nephrectomy the preferred approach in xanthogranulomatous pyelonephritis? Urology. 2006;68:952-5.

24. Benchekroun TS, Kriouil A, Belkacem A, Jorio-Benkhraba M, el Fakir Y, Benhammou M, et al. Urogenital tuberculosis in children]. Arch Pediatra. 1997;4:857-61.

25. Burghele $T$. The place of total nephro-ureterectomy in the treatment of urinary tuberculosis. Rev Chir Oncol Radiol O R L Oftalmol Stomatol Chir. 1976;25:81-8.

Correspondence address: Nianzeng Xing, MD, PhD Professor of the Department of Urology, and the Vice President of Beijing Chao-Yang Hospital, Capital Medical University Fax: + 1065 935-241 E-mail: xingnianzeng@hotmail.com 